\title{
Restoring ergodicity of stochastically reset anomalous-diffusion processes
}

\author{
Wei Wang $\odot,{ }^{1,2,{ }^{*}}$ Andrey G. Cherstvy $\odot,{ }^{2,3, \dagger}$ Ralf Metzler $\odot,{ }^{2, \ddagger}$ and Igor M. Sokolov $\odot^{3,4, \S}$ \\ ${ }^{1}$ Max Planck Institute for the Physics of Complex Systems, Nöthnitzer Straße 38, 01187 Dresden, Germany \\ ${ }^{2}$ Institute of Physics \& Astronomy, University of Potsdam, Karl-Liebknecht-Straße 24/25, 14476 Potsdam-Golm, Germany \\ ${ }^{3}$ Institut für Physik, Humboldt-Universität zu Berlin, Newtonstraße 15, 12489 Berlin, Germany \\ ${ }^{4}$ IRIS Adlershof, Zum Großen Windkanal 6, 12489 Berlin, Germany
}

(Received 17 November 2021; accepted 26 January 2022; published 28 February 2022)

\begin{abstract}
How do different reset protocols affect ergodicity of a diffusion process in single-particle-tracking experiments? We here address the problem of resetting of an arbitrary stochastic anomalous-diffusion process (ADP) from the general mathematical points of view and assess ergodicity of such reset ADPs for an arbitrary resetting protocol. The process of stochastic resetting describes the events of the instantaneous restart of a particle's motion via randomly distributed returns to a preset initial position (or a set of those). The waiting times of such resetting events obey the Poissonian, Gamma, or more generic distributions with specified conditions regarding the existence of moments. Within these general approaches, we derive general analytical results and support them by computer simulations for the behavior of the reset mean-squared displacement (MSD), the new reset increment-MSD (iMSD), and the mean reset time-averaged MSD (TAMSD). For parental nonreset ADPs with the $\operatorname{MSD}(t) \propto t^{\mu}$ we find a generic behavior and a switch of the short-time growth of the reset iMSD and mean reset TAMSDs from $\propto \Delta^{\mu}$ for subdiffusive to $\propto \Delta^{1}$ for superdiffusive reset ADPs. The critical condition for a reset ADP that recovers its ergodicity is found to be more general than that for the nonequilibrium stationary state, where obviously the iMSD and the mean TAMSD are equal. The consideration of the new statistical quantifier, the iMSD - as compared to the standard MSD—restores the ergodicity of an arbitrary reset ADP in all situations when the $\mu$ th moment of the waiting-time distribution of resetting events is finite. Potential applications of these new resetting results are, inter alia, in the area of biophysical and soft-matter systems.
\end{abstract}

DOI: 10.1103/PhysRevResearch.4.013161

\section{INTRODUCTION}

\section{A. Stochastic resetting and its applications}

Stochastic processes under restart have attracted scientific interest in the statistical-physics community in recent years [1-4], gaining considerable momentum recently due to a series of theoretical works [5-23] which uncover a number of nonintuitive features of various resetting strategies. First, resetting events drive the system out of "equilibrium" even though the stochastic process may attain a "stationary state" at long times, the so-called nonequilibrium stationary state (NESS). The second feature is that the resetting protocol may enhance search efficiency in certain first-passage problems.

Resetting has a wide range of potential applications in (nonequilibrium) thermodynamics [24,25], in the description of chemical reactions [26,27], for extreme-value statistics [28,29], behavior of complex networks [30,31], and in optimal-search problems $[9,14,15]$. The concepts of stochastic

\footnotetext{
*weiwangnuaa@gmail.com

†a.cherstvy@gmail.com

†rmetzler@uni-potsdam.de

§igor.sokolov@physik.hu-berlin.de
}

Published by the American Physical Society under the terms of the Creative Commons Attribution 4.0 International license. Further distribution of this work must maintain attribution to the author(s) and the published article's title, journal citation, and DOI. resetting are, inter alia, applicable to (for brevity, without explicit references here) (i) optimization of generic problems with "'returns," (ii) mathematical description of foraging strategies employed by animals and their movement-ecology data, (iii) stochastic-switching mechanisms between different phenotypes in a simple organism, (iv) certain classes of computational search-and-recognition algorithms, (v) description of principles of eye movements upon perception of art paintings and images, (vi) interrupted/manipulated diffusion of micron-sized beads in optical traps, and (vii) modeling of prices of reset- and barrier-type options in financial mathematics.

\section{B. Anomalous diffusion, two types of statistical averaging, and the concept of ergodicity}

Anomalous-diffusion processes (ADPs) under various stochastic-resetting protocols, intensively studied recently [10-13,19-22,32-39], bring up potential applications, e.g., for the description of data stemming from single-particletracking experiments [40] in the area of biophysics, soft-matter physics, biochemistry, and (superresolution-) microscopy imaging. Mathematically, ADPs feature a powerlaw growth of the mean-squared displacement (MSD) with time [41-44],

$$
\left\langle x^{2}(t)\right\rangle=2 K_{\mu} t^{\mu} .
$$

The MSD exponent $\mu$ classifies different subclasses of ADPs: we distinguish subdiffusion for $0<\mu<1$, superdiffusion for $1<\mu<2$, and hyperdiffusion for $\mu>2$. 
Often, however, in single-particle-tracking experiments, the particle trajectories are not numerous and "uniform enough"-regarding the "environmental conditions" of motion, dispersion of the tracer sizes, distribution of observation times, etc. [45] - to construct a satisfactory statistical ensemble for ensemble-based averaging (1). Often fewer but long time series $x_{i}(t)$ are recorded so that the concept of the time-averaged MSD (TAMSD) is more appropriate for their quantitative statistical analysis.

The TAMSD is commonly defined as the sliding average of the squared increments along a single trajectory of the $i$ th particle of length $T$, namely $[42,44]$

$$
\overline{\delta_{i}^{2}(\Delta, T)}=\frac{1}{T-\Delta} \int_{0}^{T-\Delta}\left[x_{i}(t+\Delta)-x_{i}(t)\right]^{2} d t,
$$

where $\Delta$ is the lag time. The concept of ergodicity we implement below_-first introduced by Boltzmann [46]—implies the MSD-to-TAMSD equivalence in the limit of short lag times and sufficiently long (ideally, infinite) trajectories, adequately sampling the entire phase space and visiting during time $T$ the phase-space cells in an infinitesimal proximity to any chosen cell, so that

$$
\lim _{\Delta / T \rightarrow 0} \overline{\delta_{i}^{2}(\Delta, T)}=\left\langle x^{2}(\Delta)\right\rangle .
$$

Averaging over $N$ realizations of a fluctuating variable $\overline{\delta_{i}^{2}(\Delta)}$, the so-called mean TAMSD at a given value of $\Delta$ is evaluated from the already computed TAMSDs (2) as an ensemble average,

$$
\overline{\left\langle\overline{\delta^{2}(\Delta)}\right\rangle}=\frac{1}{N} \sum_{i=1}^{N} \overline{\delta_{i}^{2}(\Delta)} .
$$

The degree of reproducibility of individual TAMSD trajectories from one realization of the process to the next is, in addition to the MSD-to-TAMSD equivalence prescribed by Eq. (3), another characteristic feature of ergodic diffusion [44] both for experimental and in silico generated time series. Hereafter, the angular brackets denote ensemble averaging, while time-averaged quantities are supplemented with the overline.

\section{Example of reset fractional Brownian motion}

As a concrete example of a stochastic process [47], both normal and ADPs [42-44] can be considered, with a single or probabilistically distributed reset points [18] and with a preset distribution of waiting times between the return events. For instance, fractional Brownian motion (FBM) is a paradigmatic ergodic ADP $[48,49]$ widely employed to describe experimental observations of anomalous diffusion on different lengthand timescales. The question we want to address is how various resetting protocols affect ergodicity of FBM and other ADPs. The TAMSD of FBM under Poissonian resetting with the waiting-time density (WTD)

$$
\phi(t)=\theta^{-1} e^{-t / \theta}
$$

of resetting events-that is the most widely used protocol in the resetting literature [18]—was first derived in Ref. [20]. The asymptotic growth of the TAMSD at short lag times
$\Delta \ll \theta \ll T$ was obtained, based on the results of systematic computer simulations of Ref. [20], in the form

$$
\left\langle\overline{\delta_{\text {reset }}^{2}(\Delta, T)}\right| \approx 2 K_{\mu}\left[\Delta^{\mu}+(\Delta / \theta)^{1} \theta^{\mu} \Gamma(\mu+1)\right] .
$$

Hereafter $\theta$ denotes the mean reset-waiting time. To distinguish the physical quantities of reset processes we denote them by the subscript "reset" in Eq. (6) and hereafter. Thus, at short lag times the $\left\langle\overline{\delta_{\text {reset }}^{2}(\Delta)}\right\rangle$ has two distinct leadingorder regimes, switching from $\propto \Delta^{\mu}$ growth for subdiffusive to $\propto \Delta^{1}$ growth for superdiffusive parental ADPs. Thus, the nonequivalence of the reset MSD

$$
\left\langle x_{\text {reset }}^{2}(t)\right\rangle \approx 2 K_{\mu} \Delta^{\mu}
$$

and the reset mean TAMSD (6) at short times was revealed for superdiffusive FBM, indicative of weak ergodicity breaking $[42,44]$; see also Sec. II C. At long times, in the NESS, the plateau of $\left\langle\overline{\delta_{\text {reset }}^{2}(\Delta)}\right\rangle$ for reset FBM was shown to be twice that of the reset MSD [20].

\section{Problem formulation and concept of iMSD}

Two natural general questions arise. First, does the existence of such two distinct exponents and switching of the TAMSD growth for sub- to superdiffusion also stay the same for other reset ADPs, beyond the model of reset FBM [20]? Second, what is the effect of the actual resetting protocol used-with different WTDs implemented, such as Gamma, Poissonian, power-law, etc.- - and what is the mean TAMSD limit for rare resetting? The latter situation is possible experimentally when the length of the observed time series is considerably shorter than the [finite] mean reset time for a given WTD. In particular, if a given reset ADP does attain the NESS, how does stationarity affect nonergodicity of reset trajectories?

For a stationary reset ADP the ergodicity is discussed below in terms of the new concept of the increment-MSD (iMSD) defined as

$$
\operatorname{iMSD}(t, \Delta)=\left\langle\left(x_{\text {reset }}(t+\Delta)-x_{\text {reset }}(t)\right)^{2}\right\rangle,
$$

where time $t$ is located in the NESS region of the process. Moreover, for a reset ADP which has not yet reached the NESS, what is the critical condition for having or restoring the ergodicity?

Additionally, computing the mean TAMSD of a stochastically reset ADP via integrating the iMSD (8) can often be difficult. We thus present below an alternative way to avoid such complicated calculations and obtain an approximate expression for the mean reset TAMSD in the limit of short lag times. This limit — where the statistics of time-averaging along a single time series is most reliable [44]-is the focus of numerous single-molecule-tracking experiments.

\section{E. WTDs and survival probabilities}

We start by introducing the basic concepts used below. A reset is a sudden transition to a single preselected position; below this position is chosen $x_{\text {reset }}=x_{0}=0$ for simplicity. The probability-density function (PDF) of waiting times between two successive reset events is denoted by $\phi(t)$. If the first moment of reset WTD $\phi(t)$ exits, the mean reset-waiting 
time is given by

$$
\langle t\rangle=\int_{0}^{\infty} t^{\prime} \phi\left(t^{\prime}\right) d t^{\prime} .
$$

The survival probability-namely, the probability of no resetting event occurring until time $t$-is expressed as

$$
\Phi(t)=1-\int_{0}^{t} \phi\left(t^{\prime}\right) d t^{\prime}=\int_{t}^{\infty} \phi\left(t^{\prime}\right) d t^{\prime} .
$$

The reset rate or the density of renewals at time $t$ is defined as [10,38]

$$
\kappa(t)=\sum_{n=1}^{\infty} \phi_{n}(t),
$$

where $\phi_{n}(t)$ is the PDF of time of the $n$th resetting event,

$$
\phi_{n}(t)=\int_{0}^{t} \phi_{n-1}\left(t^{\prime}\right) \phi\left(t-t^{\prime}\right) d t^{\prime} .
$$

In particular, if the reset rate is independent of time-it can, in general, be a function of the parameters of a given resetting protocol-one gets $\kappa(t)=\kappa$.

In this work, we derive the general formulas for stochastically reset ADPs for arbitrary resetting protocols and demonstrate the iMSD-TAMSD similarity in the case of frequent resetting (finite resetting times) under a weaker and more general condition than that for the NESS. We verify these theoretical computations via performing computer simulations for the two prototypical resetting scenarios involving the Gamma- and the power-law WTD of resetting events, see Sec. IV.

Specifically, the distribution of waiting times for the Gamma-WTD resetting with the parameters $\alpha$ and $\theta$ is

$$
\phi(t)=\frac{t^{\alpha-1} e^{-t / \theta}}{\Gamma(\alpha) \theta^{\alpha}},
$$

with the reset rate independent of time $\kappa(t)=(\alpha \theta)^{-1}$. Here $\Gamma(\alpha)$ is the Gamma function and $\theta$ is the characteristic time of Eq. (13). The situation of Poissonian resetting,

$$
\phi(t)=\theta^{-1} e^{-t / \theta},
$$

is a special case of the Gamma protocol (13) at $\alpha=1$.

For power-law resetting, the WTD with the parameters $\beta$ and $\rho$ has the form

$$
\phi(t)=\frac{\beta / \rho}{(1+t / \rho)^{1+\beta}},
$$

where $\rho$ is a characteristic time. For $\beta>1$ the mean resetwaiting time is finite and the reset rate is independent of time, $\kappa(t)=(\beta-1) / \rho$. In contrast, for $0<\beta<1$ the waiting time has an infinite mean and the reset rate is time-dependent, namely, $\kappa(t)=t^{\beta-1} \rho^{-\beta} /[\Gamma(\beta) \Gamma(1-\beta)]$.

\section{F. Plan of the paper}

In this paper, we address these problems from conceptual viewpoints. The first goal is to derive the general formulas for the iMSD with the renewal approach. To obtain the respective TAMSD, one option would be to integrate the iMSD over time straightforwardly (that is, however, not possible in general due to potentially complex forms of the underlying reset
WTD). Alternatively, we propose a timescale-decomposition approach to obtain the approximate mean-TAMSD expression in the limit of short lag times. This enables us to reveal the generic form of the mean TAMSD, with a switching between the two exponents for arbitrary stationary-increment ADPs under the conditions of stochastic resetting with arbitrary reset WTDs. In particular, the ergodicity of reset ADPs in the NESS as well as at the conditions of a rare-resetting setup is discussed. In the current work, we use the Gammaand power-law-based reset WTDs and verify the analytical theoretical results by presenting their close agreement with the results of stochastic computer simulations.

The (rest of the) paper is organized as follows. In Sec. II we present the renewal-approach results for the iMSD. In Sec. III we propose the general timescale-decomposition approach to obtain the mean TAMSD for any ADP and any resetting protocol. The phenomenon of "restoration of ergodicity" in terms of the iMSD-to-TAMSD correspondence-as compared with the formerly obtained [20] MSD-to-TAMSD nonequivalence for reset FBM given by Eqs. (6) and (7)-for frequent resetting and the switching behaviors of the scaling exponents for rare resetting of general ADPs is also discussed. In Sec. IV, the examples for reset FBM are presented and the general analytical formulas are demonstrated to agree closely with the results of simulations. In Sec. $\mathrm{V}$ the conclusions are drawn and further discussions are presented.

\section{RENEWAL APPROACH FOR THE iMSD}

\section{A. General formula}

The statistics of increments of a reset ADP $x_{\text {reset }}(t)$ given by the iMSD (8) are calculated here using the renewal approach-first proposed in Refs. [2,47] and developed to resetting-containing problems in Ref. [9] — for an arbitrary type of the resetting WTD. We consider two time points $t_{1,2}$ separated by time $\Delta=\Delta_{12}=t_{2}-t_{1}$, with the positions of the reset walk $x_{\text {reset }}\left(t_{1}\right)$ and $x_{\text {reset }}\left(t_{2}\right)$, and calculate $\left\langle\left(x_{\text {reset }}\left(t_{2}\right)-x_{\text {reset }}\left(t_{1}\right)\right)^{2}\right\rangle$, where the average is taken over all possible realizations of the ADP for the fixed time instances $t_{1}$ and $t_{2}$ (the standard ensemble-based average, with no sampling of resetting events being performed). Let us introduce three additional times: $t_{-}$is the time of the last renewal preceding $t_{1}, t_{f}$ is the time of the first renewal after $t_{1}$, and $t_{+}$is the time of the last renewal preceding $t_{2}$. Let us use $\Delta_{1}=t_{1}-t_{-}$ and $\Delta_{2}=t_{2}-t_{+}$as the times elapsed from the last renewal and prior to the measurement. We use here $\Delta=\Delta_{12}$ for simplicity: $\Delta_{12}$ is related to time $t_{1}$ that can be nonzero, as compared to the lag time $\Delta$ in the TAMSD related to the very start of the time series, $t=0$.

Three situations $(a, b$, and $c)$ for the iMSD as a function of $R_{a, b, c}$-covering all possible arrangements of the reset times for given values of $t_{1}$ and $t_{2}$ - are to be distinguished, as illustrated in Fig. 1. In the first case (a) $t_{f}>t_{2}$, i.e., there were no renewals between $t_{1}$ and $t_{2}$, wherein $t_{+}$will coincide with $t_{-}$. Then the increment of $x_{\text {reset }}(t)$ between $t_{1}$ and $t_{2}$ is that of the pure displacement process featuring stationary increments. Its mean square, conditioned on the corresponding event, is

$$
R_{a}=\left\langle\left(x_{\text {reset }}\left(t_{2}\right)-x_{\text {reset }}\left(t_{1}\right)\right)^{2}\right\rangle=2 K_{\mu} \Delta^{\mu} .
$$


(a)

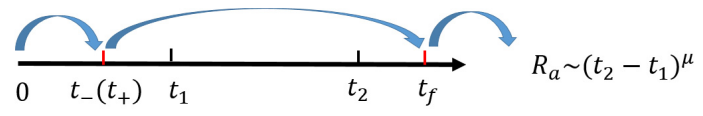

(b)

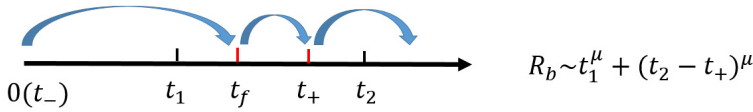

(c)

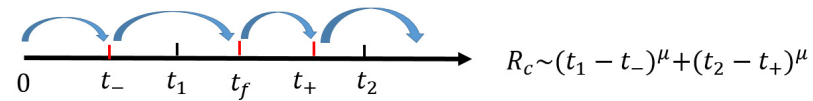

FIG. 1. Schematic of three situations for the iMSD (8) (denoted by the function $\left.R_{a, b, c}\right)$. Three characteristic reset timings $t_{-}, t_{+}$, and $t_{f}$ are labeled by the red ticks.

The probability of such a situation is denoted as

$$
P_{a}\left(t_{1}, t_{2}\right)=\int_{t_{2}}^{\infty} p_{a}\left(t_{f} \mid t_{1}\right) d t_{f},
$$

where $p_{a}\left(t_{f} \mid t_{1}\right)$ is the PDF of $t_{f}$ conditioned on $t_{1}$,

$$
p_{a}\left(t_{f} \mid t_{1}\right)=\phi\left(t_{f}\right)+\int_{0}^{t_{1}} \kappa\left(t_{-}\right) \phi\left(t_{f}-t_{-}\right) d t_{-} .
$$

The first term in Eq. (18) accounts for the case of no resetting occurring before time $t_{1}$, while the second one accounts for the last renewal taking place at $t_{-}$before $t_{1}$. The probability that $t_{f}$ is larger than $t_{2}$ is then

$$
\begin{aligned}
P_{a}\left(t_{1}, t_{2}\right) & =\int_{t_{2}}^{\infty} p\left(t_{f} \mid t_{1}\right) d t_{f} \\
& =\int_{t_{2}}^{\infty} \phi\left(t_{f}\right) d t_{f}+\int_{t_{2}}^{\infty} d t_{f} \int_{0}^{t_{1}} \kappa\left(t_{-}\right) \phi\left(t_{f}-t_{-}\right) d t_{-} \\
& =\Phi\left(t_{2}\right)+\int_{0}^{t_{1}} d t_{-} \kappa\left(t_{-}\right) \Phi\left(t_{2}-t_{-}\right),
\end{aligned}
$$

where $\Phi(t)$ is given by Eq. (10).

The case (b) in Fig. 1 corresponds to at least one renewal between $t_{1}$ and $t_{2}$ and there were no renewals prior to $t_{1}$. The coordinate of the walker at $t_{1}$ is $x_{\text {reset }}\left(t_{1}\right)=x\left(t_{1}\right)$ and its position at $t_{2}$ is $x_{\text {reset }}\left(t_{2}\right)=x\left(t_{2}-t_{+}\right)$. For fixed times $t_{1}$ and $t_{2}$, these two coordinates are independent random variables. The iMSD (8) for case (b) then is

$$
R_{b}=2 K_{\mu}\left[t_{1}^{\mu}+\left(t_{2}-t_{+}\right)^{\mu}\right]=2 K_{\mu}\left(t_{1}^{\mu}+\Delta_{2}^{\mu}\right) .
$$

To obtain the corresponding PDF for the case (b), we consider two situations: If $t_{+}=t_{f}$ then there is only one reset event between $t_{1}$ and $t_{2}$, whereas if $t_{+} \neq t_{f}$ then more than one resetting takes place within the same period. Therefore, the conditioned PDF corresponding to $R_{b}$ is

$$
\begin{aligned}
& p_{b}\left(t_{+} \mid t_{1}, t_{2}\right)=\phi\left(t_{+}\right) \Phi\left(t_{2}-t_{+}\right) \\
& \quad+\int_{t_{1}}^{t_{+}} \phi\left(t_{f}\right) \kappa\left(t_{+}-t_{f}\right) \Phi\left(t_{2}-t_{+}\right) d t_{f} .
\end{aligned}
$$

The third case (c) illustrated in Fig. 1 is when there was at least one renewal between times $t_{1}$ and $t_{2}$ and $t_{-} \neq 0$. Then the coordinate of the walker at $t_{1}$ is $x_{\text {reset }}\left(t_{1}\right)=x\left(t_{1}-t_{-}\right)$and its position at $t_{2}$ is $x_{\text {reset }}\left(t_{2}\right)=x\left(t_{2}-t_{+}\right)$. The iMSD of this case is

$$
R_{c}=2 K_{\mu}\left[\left(t_{1}-t_{-}\right)^{\mu}+\left(t_{2}-t_{+}\right)^{\mu}\right]=2 K_{\mu}\left(\Delta_{1}^{\mu}+\Delta_{2}^{\mu}\right)
$$

and the corresponding conditional PDF becomes

$$
\begin{aligned}
& p_{c}\left(t_{-}, t_{+} \mid t_{1}, t_{2}\right)=\kappa\left(t_{-}\right) \phi\left(t_{+}-t_{-}\right) \Phi\left(t_{2}-t_{+}\right)+\int_{t_{1}}^{t_{+}} \kappa\left(t_{-}\right) \\
& \times \phi\left(t_{f}-t_{-}\right) \kappa\left(t_{+}-t_{f}\right) \Phi\left(t_{2}-t_{+}\right) d t_{f} .
\end{aligned}
$$

Using the iMSDs (16), (20), and (22) as well as (19), (21), and (23), we arrive at the general expression for the iMSD

$$
\begin{aligned}
& \left\langle\left(x_{\text {reset }}\left(t_{2}\right)-x_{\text {reset }}\left(t_{1}\right)\right)^{2}\right\rangle \\
& =2 K_{\mu}\left[P_{a}\left(t_{1}, t_{2}\right) \Delta^{\mu}+\int_{t_{1}}^{t_{2}} p_{b}\left(t_{+} \mid t_{1}, t_{2}\right)\left(t_{1}^{\mu}+\Delta_{2}^{\mu}\right) d t_{+}\right. \\
& \left.\quad+\int_{0}^{t_{1}} d t_{-} \int_{t_{1}}^{t_{2}} d t_{+} p_{c}\left(t_{-}, t_{+} \mid t_{1}, t_{2}\right)\left(\Delta_{1}^{\mu}+\Delta_{2}^{\mu}\right)\right]
\end{aligned}
$$

Here either the times $t_{ \pm}$or $\Delta_{1,2}$ can be considered as independent integration variables. Changing the variables, the iMSD of a reset ADP (24) can be simplified to give

$$
\begin{aligned}
& \left\langle\left(x_{\text {reset }}\left(t_{1}+\Delta\right)-x_{\text {reset }}\left(t_{1}\right)\right)^{2}\right\rangle \\
& =2 K_{\mu}\left(\Phi\left(t_{1}+\Delta\right)+\int_{0}^{t_{1}} \kappa\left(t_{1}-\Delta_{1}\right) \Phi\left(\Delta+\Delta_{1}\right) d \Delta_{1}\right) \Delta^{\mu} \\
& \quad+2 K_{\mu}\left(\Phi\left(t_{1}\right)-\Phi\left(t_{1}+\Delta\right)\right) t_{1}^{\mu}+2 K_{\mu} \int_{0}^{t_{1}} \kappa\left(t_{1}-\Delta_{1}\right) \\
& \quad \times\left(\Phi\left(\Delta_{1}\right)-\Phi\left(\Delta_{1}+\Delta\right)\right) \Delta_{1}^{\mu} d \Delta_{1} \\
& \quad+2 K_{\mu} \int_{0}^{\Delta} \kappa\left(\Delta-\Delta_{2}\right) \Phi\left(\Delta_{2}\right) \Delta_{2}^{\mu} d \Delta_{2} .
\end{aligned}
$$

Here we remind the reader that $\kappa(t)$ is the reset rate (11) and $\Phi(t)$ is the survival probability (10).

The iMSD (25) obtained by the renewal approach is our first result valid for an arbitrary reset WTD. Obviously, the iMSD is a nonstationary characteristic, depending on $t_{1}$. Except for Poissonian resetting, the general analytical computation of the iMSD (25) - for instance, for Gammaor power-law-type resetting - is complicated. The analytical iMSD and the mean TAMSD for ADPs under Poissonian resetting are provided in Sec. IV A, with the approximate results discussed there for the MSD $\left(t_{1}=0\right)$ and $\operatorname{iMSD}\left(t_{1}>0\right)$ for any form of the reset WTD.

\section{B. Case $t_{1}=0$}

The general iMSD (25) depends on the start time of the observation, $t_{1}$. For $t_{1}=0$ the iMSD reduces to the standard MSD measuring the average distance from the starting position $x_{\text {reset }}=0$, namely,

$$
\begin{aligned}
& \left\langle x_{\text {reset }}^{2}(\Delta)\right\rangle \\
& =2 K_{\mu}\left[\Phi(\Delta) \Delta^{\mu}+\int_{0}^{\Delta} \kappa\left(\Delta-\Delta_{2}\right) \Phi\left(\Delta_{2}\right) \Delta_{2}^{\mu} d \Delta_{2}\right] .
\end{aligned}
$$

The MSDs for different ADPs under resetting were intensely studied recently \{for example, for FBM [20], heterogeneous diffusion processes [20] and geometric Brownian motion $[22,50]$ under Poissonian resetting, for continuous-time random walks under power-law resetting [12,13], for (renewal and nonrenewal) scaled Brownian motion under Poissonian and power-law resetting $[10,11]\}$. Here we provide a general 
expression for the MSD of an arbitrary ADP under arbitraryWTD resetting.

The derivation procedure is as follows. We note that the convolution integral

$$
J(T)=\int_{0}^{T} \kappa\left(T-t^{\prime}\right) \Phi\left(t^{\prime}\right) d t^{\prime}
$$

can be given in the Laplace domain as

$$
\widehat{J}(s)=\widehat{\kappa}(s) \widehat{\Phi}(s)=\frac{\widehat{\phi}(s)}{1-\widehat{\phi}(s)} \frac{1-\widehat{\phi}(s)}{s}=\frac{\widehat{\phi}(s)}{s} .
$$

Hereafter, the "hat" denotes the Laplace transform,

$$
\widehat{\phi}(s)=\int_{0}^{\infty} \phi(t) e^{-s t} d t .
$$

Therefore, the integral (27) equals

$$
J(T)=\int_{0}^{T} \phi\left(t^{\prime}\right) d t^{\prime}=1-\Phi(T) .
$$

Using the expansion at short lag times,

$$
\Phi(\Delta) \approx \Phi(0)+\Phi^{\prime}(0) \Delta=1-\phi(0) \Delta,
$$

the second term in Eq. (26) can be neglected. We stress that expansion (31) - and thus the entire approximate computation procedure based on it-necessitates (i) the existence of the derivative $d \Phi(t) / d t$ at $t=0$ and (ii) requires $\phi(0) \neq 0$ (for the leading-order lag-time-dependence to be captured correctly).

The condition (i) may be violated around $t_{1}=0$, e.g., for the Gamma distribution with $\alpha<1$. Before discussing the conditions of existence of $\Phi^{\prime}(0)$, we show that at short lag times the second term can be neglected in Eq. (26). With the Laplace-transform scheme for Eq. (27) yielding expression (30), the second MSD term reads

$$
\begin{aligned}
& \int_{0}^{\Delta} \kappa\left(\Delta-\Delta_{2}\right) \Phi\left(\Delta_{2}\right) \Delta_{2}^{\mu} d \Delta_{2} \\
& \quad<\Delta^{\mu} \int_{0}^{\Delta} \kappa\left(\Delta-\Delta_{2}\right) \Phi\left(\Delta_{2}\right) d \Delta_{2}=\Delta^{\mu}(1-\Phi(\Delta)) .
\end{aligned}
$$

If $\Phi^{\prime}(0)$ exits and is nonzero, the Taylor expansion (31) works and the second term in Eq. (26) is of order $o\left(\Delta^{\mu+1}\right)$, namely, $\Delta^{\mu}[1-\Phi(\Delta)] \approx \phi(0) \Delta^{\mu+1}$. If $\Phi^{\prime}(0)$ does not exist the order of the second term depends on the parameters of a given distribution. For instance, for the Gamma distribution (13) and the corresponding survival probability $\Phi(t)=$ $1-\gamma(\alpha, t / \theta) / \Gamma(\alpha)$, the second term in Eq. (26) is of order $o\left(\Delta^{\mu+\alpha}\right)$, namely,

$$
\begin{aligned}
& \int_{0}^{\Delta} \kappa\left(\Delta-\Delta_{2}\right) \Phi\left(\Delta_{2}\right) \Delta_{2}^{\mu} d \Delta_{2}<\Delta^{\mu}(1-\Phi(\Delta)) \\
& =\Delta^{\mu} \gamma(\alpha, \Delta / \theta) / \Gamma(\alpha) \approx \Delta^{\mu+\alpha} \theta^{-\alpha} / \Gamma(\alpha+1),
\end{aligned}
$$

which at $0<\alpha<1$ can be neglected compared to the first term of the MSD (26) scaling as $\propto \Delta^{\mu}$. We thus obtain the short-lag-time MSD asymptotic law (32) for a reset ADP for the case $t_{1}=0$. For the case $t_{1} \neq 0$, the $\gamma$ requirements (i) and (ii) are satisfied unconditionally.

Therefore, for any ADP under any reset-WTD at short lag times,

$$
\left\langle x_{\text {reset }}^{2}(\Delta)\right| \approx 2 K_{\mu} \Delta^{\mu}
$$

At long lag times, in contrast, the MSD is determined by the second term in Eq. (26) (as the first term can be neglected),

$$
\left\langle x_{\text {reset }}^{2}(\Delta)\right| \approx 2 K_{\mu} \int_{0}^{\Delta} \kappa\left(\Delta-\Delta_{2}\right) \Phi\left(\Delta_{2}\right) \Delta_{2}^{\mu} d \Delta_{2} .
$$

If the reset rate is time-independent, $\kappa(t)=\kappa$, we get

$$
\left\langle x_{\text {reset }}^{2}(\Delta)\right\rangle \approx 2 K_{\mu} \kappa Q_{\mu, \Delta},
$$

where $Q_{\mu, \Delta}$ depends on the anomalous-diffusion exponent $\mu$ and lag time $\Delta$ as follows

$$
Q_{\mu, \Delta}=\int_{0}^{\Delta} s^{\mu} \Phi(s) d s .
$$

The reset ADP attains the NESS after a characteristic time (depending on a chosen reset WTD), as long as the integral $Q_{\mu, \infty}=\int_{0}^{\infty} s^{\mu} \Phi(s) d s$ converges. In the NESS, the MSD approaches a plateau (here the subscript "st" indicates stationary quantities), with the height $\left\langle x_{\text {reset }}^{2}(\Delta)\right\rangle_{\text {st }} \approx 2 K_{\mu} \kappa Q_{\mu, \infty}$. Resetting, therefore, acts similarly to potential- or interval-induced confinements [51] of a random walk and yields a saturating or "stagnating" long-time behavior of the MSD.

For any resetting protocol, at short lag times the MSD (or the iMSD taken at $t_{1}=0$ ) has the same power law as the pure/nonreset ADP, $\left\langle x^{2}(\Delta)\right\rangle=2 K_{\mu} \Delta^{\mu}$. However, for the reset ADP the NESS emerges if and only if the expression (33) is converging. In particular, for the case of reset rate independent of time, the integral $Q_{\mu, \infty}$ exists that ensures the existence of the NESS in this case.

\section{Case $t_{1} \neq 0$}

At short lag times, using the expansion of the survival probability, $\Phi\left(t_{1}+\Delta\right) \approx \Phi\left(t_{1}\right)-\phi\left(t_{1}\right) \Delta$, we get

$$
\begin{aligned}
& \left\langle\left(x_{\text {reset }}\left(t_{1}+\Delta\right)-x_{\text {reset }}\left(t_{1}\right)\right)^{2}\right\rangle \approx 2 K_{\mu} \Delta^{\mu}+o\left(\Delta^{\mu}\right) \\
& +2 K_{\mu}\left(\int_{0}^{t_{1}} \kappa\left(t_{1}-\Delta\right) \phi(s) s^{\mu} d s+\phi\left(t_{1}\right) t_{1}^{\mu}\right) \Delta^{1} .
\end{aligned}
$$

As the term $\phi\left(t_{1}\right) t_{1}^{\mu}$ can be neglected compared with the first integral in the brackets of Eq. (36), this iMSD becomes

$$
\begin{aligned}
& \left\langle\left(x_{\text {reset }}\left(t_{1}+\Delta\right)-x_{\text {reset }}\left(t_{1}\right)\right)^{2}\right\rangle \\
& \quad \approx 2 K_{\mu}\left(\Delta^{\mu}+\int_{0}^{t_{1}} \kappa\left(t_{1}-\Delta\right) \phi(s) s^{\mu} d s \times \Delta^{1}\right) .
\end{aligned}
$$

If the reset rate is time-independent, $\kappa(t)=\kappa$, this iMSD expression turns into

$$
\left\langle\left(x_{\text {reset }}\left(t_{1}+\Delta\right)-x_{\text {reset }}\left(t_{1}\right)\right)^{2}\right\rangle \approx 2 K_{\mu}\left(\Delta^{\mu}+\kappa M_{\mu, t_{1}} \Delta^{1}\right),
$$

where $M_{\mu, t_{1}}$ is the following integral

$$
M_{\mu, t_{1}}=\int_{0}^{t_{1}} s^{\mu} \phi(s) d s .
$$

If it converges after time $t_{1}$ (that is much longer than a characteristic time), then $M_{\mu, \infty}=\int_{0}^{\infty} s^{\mu} \phi(s) d s$. We note that the iMSD for a reset ADP-at short lag times and at long enough times $t_{1}$ (depending on the time-scale of the reset WTD) - has two distinct exponents which are switching from $\propto \Delta^{\mu}$ to $\propto \Delta^{1}$ for subdiffusive and superdiffusive nonreset ADPs. Obviously, for a fixed $\mu$ and constant reset rate, the convergence of $Q_{\mu, t_{1}}$ at long times ensures the convergence of 
$M_{\mu, t_{1}}$. This implies that a reset ADP attaining the NESS has a stationary (independent of $t_{1}$ ) iMSD in the limit of short lag times $\Delta$.

The iMSD (37) at short lag times is our second result. We find the existence of two distinct growth exponents, switching in the leading order from $\propto \Delta^{1}$ to $\propto \Delta^{\mu}$ for superdiffusive and subdiffusive ADPs under a general process of resetting.

\section{TIMESCALE-DECOMPOSITION APPROACH FOR THE TAMSD}

\section{A. General formula}

We consider a time series of a resetting ADP, denoted $x_{i \text {,reset }}(t)$, with the trajectory length $T$ and discretize it into $T / \delta t$ intervals with the time step $\delta t$. The resetting events for the $i$ th trajectory-when the random walk visits the original position $x_{0}=0$-occur instantly at times $t_{i, k}=t_{k}$, with the index $k=1, \ldots, m_{i}$, consecutively after a waiting time $\tau_{i, k}=\tau_{k}=t_{k}-t_{k-1}-\delta t$. Here $m_{i}$ is the number of resetting events for the $i$ th trajectory. The strategy of the timescaledecomposition approach for computing the TAMSD is first to divide the path into independent intervals according to the successive reset-waiting time and then to collect the magnitudes of the squared increments of the reset ADP at times of resetting, $\sum_{k=1}^{m_{i}}\left[x_{i \text {,reset }}\left(t_{k}\right)-x_{i \text {,reset }}\left(t_{k}-\Delta\right)\right]^{2}$. Under the condition that the lag time is much shorter than the mean resetwaiting time (if the resetting protocol has an infinite waiting time, we set $\langle t\rangle=\infty)$ and shorter than the trace length, $\Delta \ll$ $\{\langle t\rangle, T\}$, we discretize the definition of the TAMSD to get at short lag times (for simplicity, we set $\Delta=\delta t$ below) for the $i$ th trajectory that

$$
\begin{aligned}
\overline{\delta_{i, \text { reset }}^{2}(\Delta, T)} \\
=\frac{1}{T-\Delta} \int_{0}^{T-\Delta}\left(x_{i, \text { reset }}(t+\Delta)-x_{i, \text { reset }}(t)\right)^{2} d t \\
=\frac{\Delta}{T-\Delta}\left[\sum_{k=1}^{m_{i}} \sum_{t=t_{k-1}+\Delta}^{t_{k}-\Delta}\left(x_{i, \text { reset }}(t)-x_{i, \text { reset }}(t-\Delta)\right)^{2}\right. \\
\quad+\sum_{t=t_{m_{i}}+\Delta}^{T}\left(x_{i, \text { reset }}(t)-x_{i, \text { reset }}(t-\Delta)\right)^{2} \\
\left.\quad+\sum_{k=1}^{m_{i}}\left(x_{i, \text { reset }}\left(t_{k}\right)-x_{i, \text { reset }}\left(t_{k}-\Delta\right)\right)^{2}\right]
\end{aligned}
$$

The first two terms in (40) correspond to a path of the pure ADP between the consecutive reset times. Namely, if the ADP is ergodic [44], the sum of the squared increments between the consecutive reset times is

$$
\sum_{t=t_{k-1}+\Delta}^{t_{k}-\Delta}\left(x_{i, \text { reset }}(t)-x_{i, \text { reset }}(t-\Delta)\right)^{2} \Delta \approx 2 K_{\mu} \Delta^{\mu} \tau_{k} .
$$

The third term in the reset TAMSD (40) is the collection of the iMSDs at reset times, amounting to

$$
\left(x_{i, \text { reset }}\left(t_{k}\right)-x_{i, \text { reset }}\left(t_{k}-\Delta\right)\right)^{2}=x_{i}^{2}\left(\tau_{k}\right)=x_{i, k}^{2}\left(\tau_{i, k}\right) .
$$

The right-hand side of Eq. (42) describes the free, nonreset diffusion of duratition $\tau_{i, k}$ starting from the reset time $t_{i, k}$, which are building sets of specific values for the partial trajectories $x_{i, k}$. Substituting Eqs. (41) and (42) into (40) we get the approximate TAMSD of the reset ADP at short lag times in the form

$$
\begin{aligned}
\overline{\delta_{i, \text { reset }}^{2}(\Delta, T) \approx} & \frac{2 K_{\mu}}{T-\Delta}\left(\sum_{k=1}^{m_{i}} \tau_{k}+\left(T-t_{m_{i}}\right)\right) \Delta^{\mu} \\
& +\frac{\Delta}{T-\Delta} \sum_{k=1}^{m_{i}} x_{i}^{2}\left(\tau_{k}\right) \\
\approx & 2 K_{\mu} \frac{T-m_{i} \Delta}{T} \Delta^{\mu}+\left(\frac{\Delta}{T}\right)^{1} \times \sum_{k=1}^{m_{i}} x_{i}^{2}\left(\tau_{k}\right) .
\end{aligned}
$$

Averaging over the realizations of the ADP and neglecting small terms, we ultimately arrive at

$$
\left.\overline{\left\langle\delta_{\text {reset }}^{2}(\Delta, T)\right.}\right\rangle \approx 2 K_{\mu} \Delta^{\mu}+\left\langle\sum_{k=1}^{m_{i}} x_{i}^{2}\left(\tau_{k}\right)\right\rangle\left(\frac{\Delta}{T}\right)^{1} .
$$

Here $N$ is the number of traces, the times $\tau_{k}$ contain information about the resetting events, and $\langle m\rangle=N^{-1} \sum_{i=1}^{N} m_{i}$ (to be used below). The expression (44) for the mean reset TAMSD at short lag times is our third result that is also mathematically the hardest to derive.

Some remarks are in place here. The mean TAMSD in the limit of short lag times, Eq. (44), has two distinct exponents, switching the signature behaviors from $\propto \Delta^{\mu}$ to $\propto \Delta^{1}$, the same as for the iMSD in Eqs. (37) and (38). The behavior (44) is valid for all resetting protocols, including those with infinite average reset-waiting times, $\langle t\rangle=\infty$. If the lag time is longer than the time step $\delta t$, but still well shorter than the mean resetwaiting time, $\Delta \ll\langle t\rangle$, the derived TAMSD expression (44) is still applicable. In the following, we discuss the detailed forms of the mean TAMSD when the mean reset-waiting time is finite (the reset rate is independent of time).

\section{B. Frequent resetting: iMSD restores ergodicity}

Here we focus on the situation when the mean resetwaiting time is much shorter than the measurement time,

$$
T \gg\langle t\rangle .
$$

As the mean TAMSD is the sum over independent realizations of the process, the sum in Eq. (44) can be written as

$$
\begin{aligned}
\left\langle\sum_{k=1}^{m_{i}} x_{i}^{2}\left(\tau_{k}\right)\right\rangle & =\frac{1}{N} \sum_{i=1}^{N} \sum_{k=1}^{m_{i}} x_{i}^{2}\left(\tau_{k}\right) \\
& =\frac{\sum_{k=1}^{m_{1}+. .+m_{N}} x_{i}^{2}\left(\tau_{k}\right)}{\sum_{j=1}^{N} m_{j}} \frac{\sum_{j=1}^{N} m_{j}}{N} .
\end{aligned}
$$

According to the central-limit theorem, with $\sum_{j=1}^{N} m_{j} \rightarrow \infty$, one gets

$$
\left\langle\sum_{k=1}^{m_{i}} x_{i}^{2}\left(\tau_{k}\right)\right\rangle \approx\left\langle x^{2}\left(\tau_{k}\right)\right\rangle\langle m\rangle=2 K_{\mu} \kappa T \int_{0}^{T} s^{\mu} \phi(s) d s .
$$


We thus note that the mean reset TAMSD of a reset ADP has two different exponents,

$$
\left\langle\overline{\delta_{\text {reset }}^{2}(\Delta, T)}\right\rangle \approx 2 K_{\mu}\left(\Delta^{\mu}+\kappa M_{\mu, T} \times \Delta^{1}\right),
$$

where $M_{\mu, T}$ is defined by Eq. (39). We emphasize that Eq. (48) is similar in form to the iMSD result (38).

Expressions (38) and (48) describe the limiting behaviors with the first leading-order terms $\propto \Delta^{1}$ and $\propto \Delta^{\mu}$. Higherorder terms are negligible for vanishing lag times, $\Delta \rightarrow 0$; for short but finite lag times, the statement about the iMSD-toTAMSD equality becomes less precise.

Obviously, as the reset MSD (32) at short times behaves always as $\left\langle x_{\text {reset }}^{2}(\Delta)\right\rangle=2 K_{\mu} \Delta^{\mu}$, the nonequivalence of the mean reset TAMSD and reset MSD emerges at short lag times for superdiffusive $(\mu>1)$ ADPs under resetting, indicating weak ergodicity breaking. However, comparing with the iMSD (38), we find that for $\mu>1$ the iMSD restores ergodicity in terms of the approximate equality of the reset iMSD and reset mean TAMSD at long initial times $t_{1} \rightarrow \infty$ only if the $\mu$ th moment $M_{\mu, \infty}$ exits, namely, $M_{\mu, \infty}<\infty$. Therefore, in this case we have at sufficiently short lag times the iMSD-to-TAMSD correspondence,

$$
\left\langle\overline{\delta_{\text {reset }}^{2}(\Delta, T)}\right\rangle=\left\langle\left(x_{\text {reset }}\left(t_{1}+\Delta\right)-x_{\text {reset }}\left(t_{1}\right)\right)^{2}\right\rangle,
$$

for time $t_{1}$ being in the NESS region, the fourth result of the study.

\section{Rare resetting: Outliers dominate the mean}

Here we focus on the reset TAMSD in the presence of rare resetting, i.e., when the trace length is much shorter than the mean reset-waiting time,

$$
T \ll\langle t\rangle .
$$

As resetting is so rare, the number of resetting events within a single trajectory is almost zero. Thus $m_{1}+\ldots+m_{N}$ does not tend to infinity any longer in $\sum_{j=1}^{N} m_{j}$. However, if the number of resets is regarded as a random variable taking the values of 0 or 1 , we can get the mean reset TAMSD from Eq. (44) as

$$
\begin{aligned}
\left\langle\overline{\delta_{\text {reset }}^{2}(\Delta, T)}\right\rangle & \approx 2 K_{\mu} \Delta^{\mu}+N^{-1} \sum_{k=1}^{N} H\left(T-\tau_{k}\right) x^{2}\left(\tau_{k}\right)\left(\frac{\Delta}{T}\right)^{1} \\
& \approx 2 K_{\mu} \Delta^{\mu}+2 K_{\mu} \int_{0}^{T} s^{\mu} \phi(s) d s\left(\frac{\Delta}{T}\right)^{1} . \quad \text { (51) }
\end{aligned}
$$

Here $H(x)$ is a Heaviside step-function (it is zero for $x<0$ and unity when $x>0$ ). Therefore, we get

$$
\left\langle\overline{\delta_{\text {reset }}^{2}(\Delta, T)}\right\rangle \approx 2 K_{\mu}\left(\Delta^{\mu}+M_{\mu, T} \times(\Delta / T)^{1}\right) .
$$

Surprisingly, the $\left\langle\overline{\delta_{\text {reset }}^{2}(\Delta)}\right\rangle$ expression features again two scaling exponents, with $\propto \Delta^{\mu}$ and $\propto \Delta^{1}$ scalings. In particular, for superdiffusive ADPs the mean reset TAMSD with rare resetting will have (in the limit of short lag times) a linear dependence on $\Delta$. This leads to a rather intriguing effect that "minorities" in the Gesamtheit of TAMSD trajectories dominate the mean-TAMSD behavior. These rare realizations with a linear-in-lag-time TAMSD change the overall long-lag-time behavior of the ensemble-averaged TAMSD even though most of the TAMSD trajectories are of a power-law form $\propto \Delta^{\mu}$ (as they do not contain resetting events). This is our fifth result.

\section{EXAMPLES}

To support the general model-free results of Secs. II and III, we consider below the examples of Poissonian- and powerlaw-type resetting and also validate the theoretical predictions by the results of computer simulations.

\section{A. Poissonian resetting}

\section{1. $i M S D$}

For Poissonian resetting the exact TAMSD can be obtained by integrating the iMSD and one can verify the validity of the approximate TAMSD obtained from the timescaledecomposition approach.

For the WTD (14), the mean reset-waiting time is $\langle t\rangle=\theta$ and the survival probability is $\Phi(t)=e^{-t / \theta}$ in Eq. (25). The iMSD for Poissonian-reset ADPs becomes

$$
\begin{aligned}
& \left\langle\left(x_{\text {reset }}\left(t_{1}+\Delta\right)-x_{\text {reset }}\left(t_{1}\right)\right)^{2}\right\rangle \\
& =2 K_{\mu}\left[e^{-\Delta / \theta} \Delta^{\mu}+\theta^{\mu} \gamma(\mu+1, \Delta / \theta)\right. \\
& \left.\quad+\left(1-e^{-\Delta / \theta}\right)\left(e^{-t_{1} / \theta} t_{1}^{\mu}+\theta^{\mu} \gamma\left(\mu+1, t_{1} / \theta\right)\right)\right],
\end{aligned}
$$

where the lower incomplete Gamma function is defined as

$$
\gamma(a, z)=\int_{0}^{z} e^{-x} x^{a-1} d x .
$$

When the initial time vanishes, $t_{1}=0$, the iMSD reduces to the MSD and follows

$$
\left\langle x_{\text {reset }}^{2}(\Delta)\right\rangle=2 K_{\mu}\left(e^{-\Delta / \theta} \Delta^{\mu}+\theta^{-1} Q_{\mu, \Delta}\right),
$$

where

$$
Q_{\mu, \Delta}=\theta^{\mu+1} \gamma(\mu+1, \Delta / \theta) .
$$

At short lag times, at $\Delta \ll \theta$, the MSD of a reset ADP has a power-law dependence similar to that for a pure ADP. At long lag times, at $\Delta \gg \theta$, the MSD attains a plateau (after the process has reached the NESS), with the plateau (index "pl") height

$$
\left\langle x_{\text {reset }}^{2}(\Delta)\right\rangle_{\mathrm{pl}} \approx 2 K_{\mu} \theta^{-1} Q_{\mu, \infty},
$$

where $Q_{\mu, \infty}=\theta^{\mu+1} \Gamma(\mu+1)$.

Second, we present the iMSD for long initial times, $t_{1} \gg \theta$, after which a general ADP attains the NESS. As in this case $\gamma\left(\mu+1, t_{1} / \theta\right) \approx \Gamma(\mu+1)$, the iMSD becomes

$$
\begin{aligned}
& \left\langle\left(x_{\text {reset }}\left(t_{1}+\Delta\right)-x_{\text {reset }}\left(t_{1}\right)\right)^{2}\right\rangle \\
& \approx 2 K_{\mu}\left[e^{-\Delta / \theta} \Delta^{\mu}+\theta^{\mu} \gamma(\mu+1, \Delta / \theta)\right. \\
& \left.\quad+\left(1-e^{-\Delta / \theta}\right) \theta^{\mu} \Gamma(\mu+1)\right] .
\end{aligned}
$$

At short lag times, when $\Delta \ll \theta$, neglecting the small terms here, the iMSD has two exponents,

$$
\left\langle\left(x_{\text {reset }}\left(t_{1}+\Delta\right)-x_{\text {reset }}\left(t_{1}\right)\right)^{2}\right\rangle \approx 2 K_{\mu}\left(\Delta^{\mu}+\theta^{-1} M_{\mu, \infty} \Delta^{1}\right),
$$




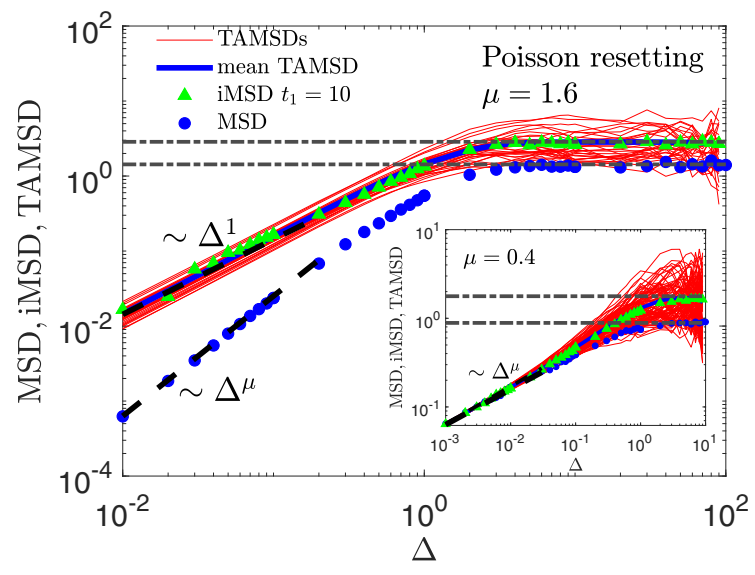

FIG. 2. FBM under the conditions of frequent Poissonian resetting [20], presented as the results of computer simulations and theoretical predictions. The reset $\operatorname{MSD}\left\langle x_{\text {reset }}^{2}(\Delta)\right\rangle$ (the blue circles), the reset iMSD $\left\langle\left(x_{\text {reset }}\left(t_{1}+\Delta\right)-x_{\text {reset }}\left(t_{1}\right)\right)^{2}\right\rangle$ (the green triangles), the individual reset TAMSD trajectories $\overline{\delta_{\text {reset }}^{2}(\Delta)}$ (the thin red curves), and the mean reset TAMSD $\left\langle\overline{\delta_{\text {reset }}^{2}(\Delta)}\right\rangle$ (the thick blue curves) are shown in the plots. The scaling relations (59) and (64) are shown as the black dashed lines. The stationary plateaus (60) are the dot-dashed lines. The main plot shows the MSD-to-TAMSD nonequivalence and the approximate equality of the iMSD to the TAMSD for superdiffusive FBM. The inset illustrates the MSDTAMSD correspondence at short lag times for subdiffusive reset FBM. We illustrate the behavior of the MSD, iMSD, and TAMSD from the minimal to the maximal lag times [including the long-lagtime regime of the NESS realized for the conditions of frequent resetting]. The scaling exponents $\mu$ are given in the legend(s). The trajectory length is $T=10^{2}$ for superdiffusive and $T=10^{1}$ for subdiffusive walks. Other parameters: the simulation time step is $d t=10^{-2}$ and $10^{-3}$ for super- and subdiffusion, respectively; the mean reset time of the WTD (14) is $\theta=1$.

where $M_{\mu, \infty}=\theta^{\mu} \Gamma(\mu+1)$. These expressions summarize the general iMSD formulas for an arbitrary ADP with the Poissonian WTD of resetting events.

\section{Example of Poissonian WTD for reset $F B M$}

In Fig. 2 we present the results of computer simulations for Poissonian-reset FBM with $\mu=1.6$ and $\mu=0.4$. The details of the numerical scheme are presented in our recent study [20] (without the iMSD concept), so we avoid its repetition here. We clearly find that for reset FBM the MSD-to-TAMSD nonequivalence for superdiffusive $\mu$ turns into the iMSDTAMSD correspondence [at short and long lag times]. This restores ergodicity of superdiffusive reset FBM in terms of this new iMSD quantifier if the process is considered after long-enough times $t_{1}$ in the NESS regime. The iMSD and the mean TAMSD are approximately equal both for superdiffusive and for subdiffusive reset FBM, both at short and long times. The previously observed [20] discrepancy in the plateau region of the stationary (index "st") NESS-related behavior,

$$
\mathrm{TAMSD}_{\text {reset, st }}=2 \times \mathrm{MSD}_{\text {reset, st }},
$$

is no longer present for the "corrected" description in terms of the iMSD, where

$$
\mathrm{TAMSD}_{\text {reset, st }} \approx \mathrm{iMSD}_{\text {reset, st }} .
$$

This statement is the sixth result of the current study; its region of validity is based on the assumptions listed after Eq. (31).

\section{TAMSD: Frequent resetting}

To verify the expression for the mean TAMSD obtained via the timescale-decomposition approach, here for the case of Poissonian resetting we integrate the iMSD over time and get the exact mean reset TAMSD as

$$
\begin{aligned}
& \left\langle\overline{\delta_{\text {reset }}^{2}(\Delta, T)}\right\rangle=2 K_{\mu}\left[e^{-\Delta / \theta} \Delta^{\mu}+\theta^{\mu} \gamma(\mu+1, \Delta / \theta)\right] \\
& +2 K_{\mu}\left(1-e^{-\Delta / \theta}\right) \theta^{\mu}[\gamma(\mu+1,(T-\Delta) / \theta) \\
& \left.+\frac{\theta}{T-\Delta}\left(\gamma\left(\mu+1, \frac{T-\Delta}{\theta}\right)-\gamma\left(\mu+2, \frac{T-\Delta}{\theta}\right)\right)\right] .
\end{aligned}
$$

When $T \gg \theta$, namely for the case of frequent resetting, the mean reset TAMSD (62) approximately equals the iMSD (58),

$$
\begin{aligned}
\left.\overline{\delta_{\text {reset }}^{2}(\Delta, T)}\right\rangle \approx & 2 K_{\mu}\left[e^{-\Delta / \theta} \Delta^{\mu}+\theta^{\mu} \gamma(\mu+1, \Delta / \theta)\right. \\
& \left.+\left(1-e^{-\Delta / \theta}\right) \theta^{\mu} \Gamma(\mu+1)\right] \\
= & \left\langle\left(x_{\text {reset }}\left(t_{1}+\Delta\right)-x_{\text {reset }}\left(t_{1}\right)\right)^{2}\right\rangle .
\end{aligned}
$$

It means that the iMSD restores ergodicity of an arbitrary Poisson-reset ADP. At short lag times, when $\Delta \ll \theta$, the reset TAMSD has two different exponents,

$$
\left\langle\overline{\delta_{\text {reset }}^{2}(\Delta, T)}\right\rangle \approx 2 K_{\mu}\left(\Delta^{\mu}+\theta^{-1} M_{\mu, \infty} \Delta^{1}\right) .
$$

The mean reset TAMSD coincides with that from the timescale-decomposition approach given by Eq. (48). We can also draw the same conclusion without exact calculations based on the critical condition for recovering the ergodicity discussed in Sec. III as the $\mu$ th moments of Poissonian resetting is finite, $M_{\mu, \infty}=\theta^{\mu} \Gamma(\mu+1)$.

\section{TAMSD: Rare resetting}

When $T \ll \theta$ the probability of reset for a single trajectory is small. The mean rarely-reset TAMSD (62) at short times, $\Delta \ll \theta$, using the relation $\gamma(y, t) \approx t^{y} / y$, becomes

$$
\left\langle\overline{\delta_{\text {reset }}^{2}(\Delta, T)}\right\rangle \approx 2 K_{\mu}\left(\Delta^{\mu}+(\mu+1)^{-1} T^{\mu}(\Delta / \theta)^{1}\right) .
$$

This mean reset TAMSD still has a linear dependence on lag time combined with a power-law dependence, indicating the short-lag-time nonequivalence of the reset mean TAMSD $\left\langle\overline{\delta_{\text {reset }}^{2}(\Delta)}\right\rangle$ and the reset MSD $\left\langle x_{\text {reset }}^{2}(\Delta)\right\rangle=2 K_{\mu} \Delta^{\mu}$ for superdiffusive Poisson-reset ADPs. Rare resetting is inherently out of equilibrium and thus no MSD-to-TAMSD equality is intuitively expected. The MSD-to-TAMSD nonequivalence therefore persists. This is our seventh result for an arbitrary rarely reset ADP. For certain experimental situations with $a$ priori unknown mean reset time, both ergodic and nonergodic dynamics and MSD-TAMSD relations might be realizable/observed at the same time (also changing as a function of the length of recorded trajectories). 


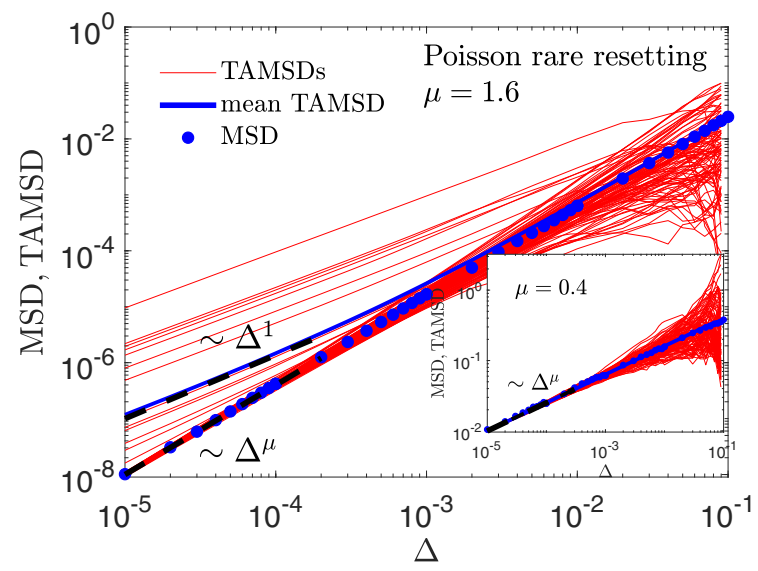

FIG. 3. Population splitting for superdiffusive FBM with rare resetting (main plot). The effect of "minorities" in the ensemble of trajectories-dominating the short-lag-time scaling of the mean TAMSD - is demonstrated. The scaling exponents of Eqs. (55) and (65) are the black dashed lines at short times. The inset illustrates reproducible realizations of subdiffusive reset FBM. The notations for the curves are the same as in Fig. 2. Parameters: $T=10^{-1}, d t=$ $10^{-5}, \theta=1$.

\section{Example of rarely reset $\mathrm{FBM}$}

The results of computer simulations of rarely reset FBM with the Hurst exponents $\mu=1.6$ and $\mu=0.4$ under Poissonian resetting are presented in the main plot and the inset of Fig. 3, respectively. In the superdiffusive case $\mu>1$ we find that at short lag times the mean reset TAMSD switches to the linear growth due to the dominant influence of a minor fraction of trajectories containing one or more events of resetting. Subdiffusive FBM with rare resets does not reveal such a splitting: fairly reproducible TAMSD trajectories are observed signifying ergodic dynamics, also featuring the MSD-TAMSD equality; see the inset of Fig. 3.

\section{B. Power-law resetting}

\section{Reset $M M S D$ and mean TAMSD}

It appears to be difficult to compute the mean TAMSD for a general ADP under power-law resetting exactly by integrating the iMSD, as we did for Poisson resetting in Sec. IV A. As the NESS is attained at $\beta>\mu+1$ (where the ergodic behavior is obviously restored [10]), we verify below the critical condition $M_{\mu, \infty}<\infty$ in the non-NESS regime of the model parameters, namely, for $1<\beta<\mu+1$.

Consider the $\mu$ th moment of reset WTD (15) with a finite mean reset time, namely

$$
M_{\mu, T}=\frac{\beta T^{1+\mu}}{(1+\mu) \rho} \int_{0}^{T / \rho} x^{\mu}(1+x)^{-(1+\beta)} d x .
$$

The integral in Eq. (66) can be expressed via the hypergeometric function ${ }_{2} F_{1}$ as follows:

$$
\int_{0}^{\frac{T}{\rho}} x^{\mu}(1+x)^{-(1+\beta)} d x={ }_{2} F_{1}(1+\mu, 1+\beta, 2+\mu,-T / \rho) .
$$

Using the Pfaff transformations [52],

$$
\begin{aligned}
{ }_{2} F_{1}(a, b, c, z) & =(1-z)^{-a}{ }_{2} F_{1}\left(a, c-b, c, \frac{z}{z-1}\right) \\
& =(1-z)^{-b}{ }_{2} F_{1}\left(b, c-a, c, \frac{z}{z-1}\right),
\end{aligned}
$$

and the representation of ${ }_{2} F_{1}$ via the Gamma functions,

$$
{ }_{2} F_{1}(a, b, c, 1)=\frac{\Gamma(c) \Gamma(c-a-b)}{\Gamma(c-a) \Gamma(c-b)},
$$

valid for $\operatorname{Re}(c)>\operatorname{Re}(a+b)$, the integral in Eq. (66) for the $\mu$ th moment will exist when $\beta>\mu$ and $T \gg \rho$, namely

$$
M_{\mu, \infty}=\beta \rho^{\mu} \Gamma(1+\mu) \Gamma(\beta-\mu) / \Gamma(1+\beta) .
$$

In the opposite case, when $\beta<\mu$ and $T \gg \rho$, the value of the integral depends on the upper integration limit $T$ as

$$
M_{\mu, T}=\beta(\mu-\beta)^{-1} \rho^{\beta} T^{\mu-\beta} .
$$

It means that if $\beta>\{\mu, 1\}$, the ergodic behavior is restored in the short-lag-time limit (with the approximate equality of the reset $\mathrm{iMSD}$ and the mean reset TAMSD),

$$
\begin{aligned}
& \left\langle\overline{\delta_{\text {reset }}^{2}(\Delta, T)}\right\rangle=\left\langle\left(x_{\text {reset }}\left(t_{1}+\Delta\right)-x_{\text {reset }}\left(t_{1}\right)\right)^{2}\right\rangle \\
& \quad=2 K_{\mu}\left(\Delta^{\mu}+\frac{\beta(\beta-1) \rho^{\mu-1} \Gamma(1+\mu) \Gamma(\beta-\mu)}{\Gamma(1+\beta)} \Delta^{1}\right) .
\end{aligned}
$$

For $1<\beta<\mu$, on the other hand, the nonequivalence of the mean reset TAMSD to the reset iMSD emerges because

$$
\left\langle\overline{\delta_{\text {reset }}^{2}(\Delta, T)}\right\rangle=2 K_{\mu}\left(\Delta^{\mu}+\frac{\beta(\beta-1) \rho^{\beta-1}}{\mu-\beta} T^{\mu-\beta} \Delta^{1}\right),
$$

while

$$
\begin{aligned}
& \left\langle\left(x_{\text {reset }}\left(t_{1}+\Delta\right)-x_{\text {reset }}\left(t_{1}\right)\right)^{2}\right\rangle \\
& =2 K_{\mu}\left(\Delta^{\mu}+\beta(\beta-1) \rho^{\beta-1}(\mu-\beta)^{-1} t_{1}^{\mu-\beta} \Delta^{1}\right),
\end{aligned}
$$

implying weak ergodicity breaking in this region of parameters.

\section{Example of power-law WTD for reset FBM}

As an example, the simulations of FBM with $\mu=1.6$ under power-law resetting with the non-NESS parameters $1<$ $\beta<1+\mu$ are presented in Fig. 4. We find that for $\beta=1.9-$ when $\beta>\mu$ giving rise to a finite $\mu$ th moment (70)- the reset iMSD and the mean reset TAMSD are approximately equal. The standard reset MSD is not equal to the mean reset TAMSD, however, as shown in Fig. 4(a). In the other case, for $\beta=1.3-$ when $\beta<\mu$ is satisfied-we find based on the results of computer simulations that the reset iMSD is not equal to the mean reset TAMSD, as our theory indeed predicts; see Fig. 4(b). Last, as intuitively expected, for the last situation of $0<\beta<1$ and thus for infinite mean reset-waiting time, the ergodicity-breaking behavior and the spread of individual 


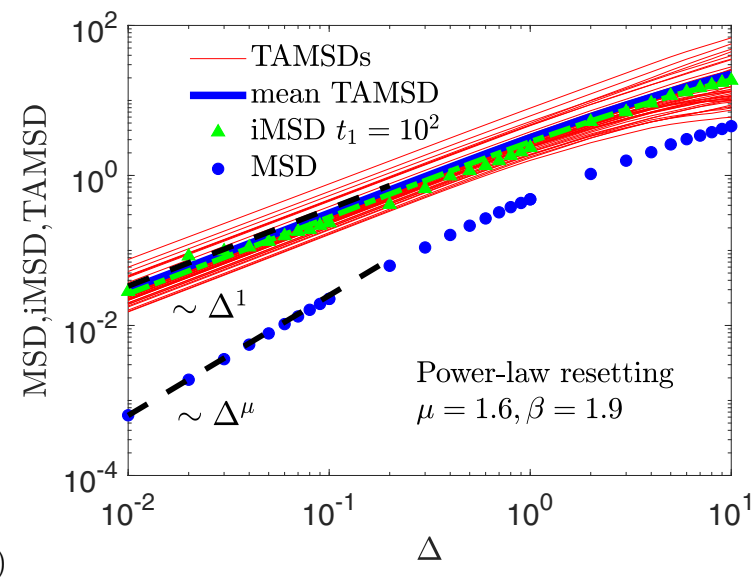

(a)

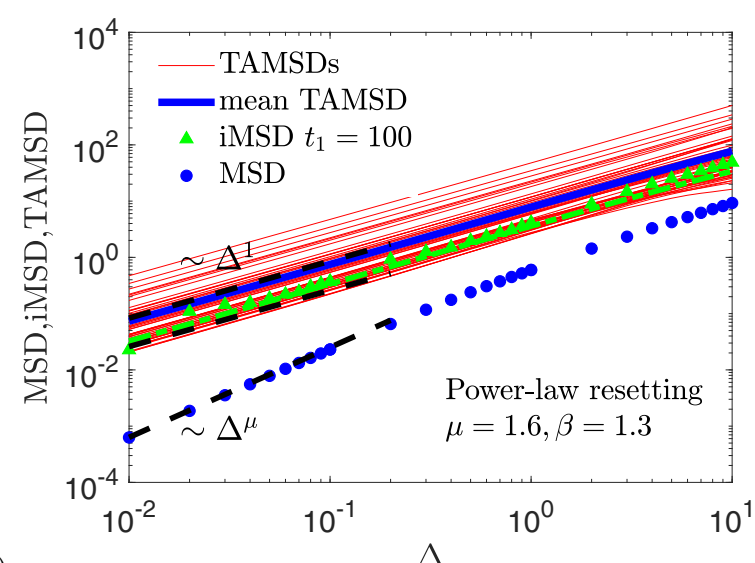

(b)

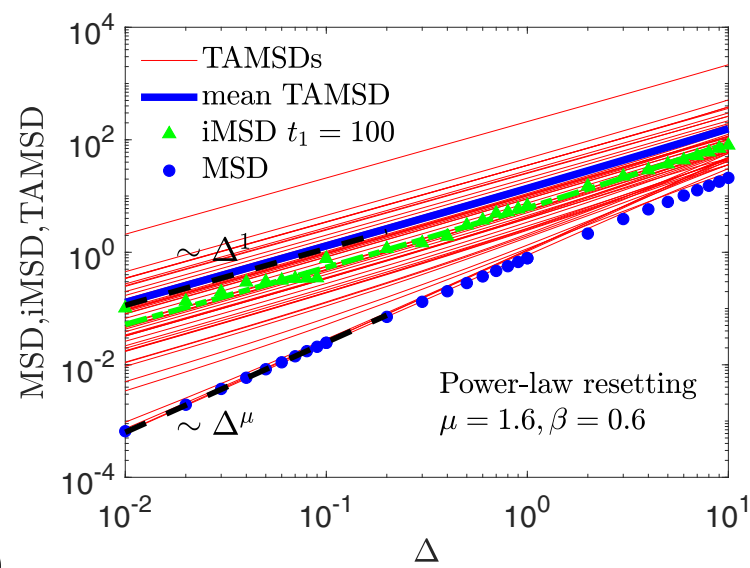

FIG. 4. Simulations of superdiffusive FBM with $\mu=1.6$ under power-law-WTD frequent resetting, computed for (a) $\beta=1.9$, (b) $\beta=1.3$, and (c) $\beta=0.6$. The scaling behaviors of $2 K_{\mu} \Delta^{\mu}$, of Eqs. (74) and (73) are shown as the black dashed asymptotes (for the case of finite reset-waiting times). For infinite mean reset-waiting times, the dashed black line for the mean TAMSD is the approximation of Eq. (48) with $\kappa$ being replaced by $\kappa(T)$. The exact iMSD (25) is shown by the green dashed line. The curves have the same notations as in Fig. 2. The plots only show the regions of short lag times. Parameters: $T=10^{3}, d t=10^{-2}, \rho=1, t_{1}=10^{2}$.

TAMSD realizations is similar to the rare-resetting scenario, with many TAMSD realizations not featuring a single event of resetting; see Fig. 4(c).

\section{CONCLUSIONS AND DISCUSSIONS}

In this work, we established a general framework to assess ergodicity-in terms of the MSD-to-TAMSD approximate equality-of arbitrary stationary-increment ADPs with the MSD of the form $\left\langle x^{2}(t)\right\rangle=2 K_{\mu} t^{\mu}$ under stochastic resetting with an arbitrary WTD. We derived the analytical expressions for the newly introduced iMSD [the first key result; Eq. (25)] and the mean TAMSD [the third main result; Eq. (44)] employing the general approach of renewal and the timescale-decomposition scheme for arbitrary resetting protocols. A characteristic switching between the two distinct growth behaviors is revealed for subdiffusive and superdiffusive nonreset ADPs, $\propto \Delta^{\mu}$ and $\propto \Delta^{1}$, for both the iMSD [the second key result, Eq. (37)] and the mean TAMSD of the reset process with no weak ergodicity breaking [the fourth main result; Eqs. (48) and (49)].

The renewal approach to the iMSD is applicable to such stationary-increments ADPs as FBM [20,53], Brownian motion, Ornstein-Uhlenbeck process [54], as well as Brownian motion with "diffusing diffusivity" [55]. The approach is not applicable to nonstationary and intrinsically ageing ADPs, such as scaled Brownian motion [10,11,56,57], heterogeneous diffusion processes [20,58], and continuous-time random walks [59-61].

In addition, we found a critical condition that if the $\mu$ th moment of the resetting WTD exits, the correspondence of the iMSD to the mean TAMSD emerges. This condition for a superdiffusive reset ADP with $\mu>1$ is $\int_{0}^{\infty} s^{\mu} \phi(s) d s<\infty$ that is an extension of a stronger NESS condition $\int_{0}^{\infty} s^{\mu} \Phi(s) d s<$ $\infty$. For Poissonian resetting they both give the same result. This means that the reset iMSD behavior observed at short times after the system attains the NESS follows exactly the same exponents and has the same magnitudes as the mean reset TAMSD in the same limit [the sixth key result; Eq. (61)].

We also derived the mean reset TAMSD for a generic ADP under the conditions of rare resetting (50) [the fifth key result; Eq. (52)]. It was demonstrated again to feature two scaling exponents, with the $\propto \Delta^{\mu}$ and $\propto \Delta^{1}$ growth, indicating the nonequivalence of the reset mean TAMSD and reset MSD in the regime of short lag times [the seventh main result; Eq. (65)]. The analytical results were verified by computer simulations of FBM ADP with rare Poissonian resetting.

The timescale-decomposition approach we employed is applicable not only to ergodic but also to nonergodic nonreset initial processes. The TAMSD of a nonergodic pure ADP under frequent resetting with a constant reset rate $\kappa$ can be obtained via employing the TAMSD with the dependence on the path length (41),

$$
\sum_{t=t_{k-1}+\Delta}^{t_{k}-\Delta}\left(x_{\text {reset }}(t)-x_{\text {reset }}(t-\Delta)\right)^{2} \Delta \approx \overline{\delta^{2}\left(\Delta, \tau_{k}\right)} \tau_{k} .
$$

Then, the mean TAMSD at short lag times is

$$
\left.\left\langle\overline{\delta_{\text {reset }}^{2}(\Delta, T)}\right\rangle \approx \kappa \int_{0}^{T}\left(\overline{\left\langle\delta^{2}(\Delta, s)\right.}\right\rangle s+\left\langle x^{2}(s)\right\rangle \Delta\right) \phi(s) d s .
$$

This approach is applicable to all ADPs. For nonreset processes with the MSD of, for instance, an exponential or logarithmic form [62], the approach works too, with the TAMSD 
at short lag times acquiring a dependence on the path length $\tau_{k}$; see Eq. (75). As an example, the short-lag-time asymptotic behavior of the mean reset TAMSD for reset geometric Brownian motion [22] can be obtained from there. This approach is also applicable for ADPs with the TAMSD at short lag times being a function of the trajectory length $T$, like for nonstationary and ageing ADPs [44] (e.g., the processes of scaled Brownian motion [10,11] and heterogeneous diffusion [20,58]).

Recently, the ergodic features of normal diffusion (with and without drift) under Poissonian resetting were examined in Ref. [63]. Via computing the autocorrelation function and the mean TAMSD, the nonergodicity of the reset process in terms of the MSD-to-TAMSD nonequivalence was assessed [63]. For drift-free normal-diffusion walks with resets, the results of Eq. (47) in Ref. [63] are identical to our Eq. (63) for generic ADPs. In contrast to Poisson-reset normal diffusion of Ref. [63], our findings are general and valid for arbitrary ADPs and any WTDs of the resetting protocol.

The information about the mean TAMSD of a reset ADP can be extracted prior to the experiment as long as we have knowledge about the statistics of the nonreset ADP and characteristics of the resetting protocol. From the experimental perspective, it would be interesting to look at ADPs with two different scaling exponents, which turn from $\mu_{1}$ to $\mu_{2}$ at a known lag time $\Delta_{12}$. This situation is ubiquitously observed in single-particle-tracking setups, with $\mu_{2}<\mu_{1}$ typically $[45,64]$. The conclusions for such a switching-diffusion scenario in the presence of stochastic resetting, at least in certain regimes, can be the goal of the future research.
The approach of the system to the NESS is an interesting problem per se; being studied in several pivotal resetting studies [18] for conventional Brownian motion, the transition regime will be of interest to investigate also for general processes of anomalous diffusion (e.g., the duration and spatial extent of this regime as a function of the exponent $\mu$, etc.). This can become a separate future investigation, beyond the scope of the current analysis.

Finally, the question about particle-particle interactionsespecially at the conditions of the NESS-is similarly appealing. Its implementation will, however, require severe modifications of the current approaches developed for diffusion and resetting of inert or noninteracting particles, including the introduction of concepts of interparticle interaction range and particle density (on a discrete lattice of potentially accessible sites).

\section{ACKNOWLEDGMENTS}

A.G.C. is grateful to Humboldt University of Berlin and to University of Potsdam for hospitality and support. R.M. acknowledges financial support by the Deutsche Forschungsgemeinschaft (DFG Grant ME 1535/12-1). R.M. thanks the Foundation for Polish Science (Fundacja na rzecz Nauki Polskiej) for support within an Alexander von Humboldt Polish Honorary Research Scholarship. Funded by the Deutsche Forschungsgemeinschaft (DFG, German Research Foundation) - Projektnummer 491466077.
[1] S. C. Manrubia and D. H. Zanette, Stochastic multiplicative processes with reset events, Phys. Rev. E 59, 4945 (1999).

[2] M. R. Evans and S. N. Majumdar, Diffusion with Stochastic Resetting, Phys. Rev. Lett. 106, 160601 (2011).

[3] M. R. Evans and S. N. Majumdar, Diffusion with optimal resetting, J. Phys. A 44, 435001 (2011).

[4] M. R. Evans, S. N. Majumdar, and K. Mallick, Optimal diffusive search: Nonequilibrium resetting versus equilibrium dynamics, J. Phys. A 46, 185001 (2013).

[5] L. Kusmierz, S. N. Majumdar, S. Sabhapandit, and G. Schehr, First Order Transition for the Optimal Search Time of Lévy Flights with Resetting, Phys. Rev. Lett. 113, 220602 (2014).

[6] S. Reuveni, Optimal Stochastic Restart Renders Fluctuations in First Passage Times Universal, Phys. Rev. Lett. 116, 170601 (2016).

[7] A. Pal and S. Reuveni, First Passage Under Restart, Phys. Rev. Lett. 118, 030603 (2017).

[8] M. R. Evans and S. N. Majumdar, Run and tumble particle under resetting: A renewal approach, J. Phys. A 51, 475003 (2018).

[9] A. V. Chechkin and I. M. Sokolov, Random Search with Resetting: A Unified Renewal Approach, Phys. Rev. Lett. 121, 050601 (2018).

[10] A. S. Bodrova, A. V. Chechkin, and I. M. Sokolov, Scaled Brownian motion with renewal resetting, Phys. Rev. E 100, 012120 (2019).

[11] A. S. Bodrova, A. V. Chechkin, and I. M. Sokolov, Nonrenewal resetting of scaled Brownian motion, Phys. Rev. E 100, 012119 (2019).
[12] A. S. Bodrova and I. M. Sokolov, Continuous-time random walks under power-law resetting, Phys. Rev. E 101, 062117 (2020).

[13] T. Zhou, P. Xu, and W. Deng, Continuous-time random walks and Lévy walks with stochastic resetting, Phys. Rev. Research 2, 013103 (2020).

[14] A. Pal, L. Kusmierz, and S. Reuveni, Search with home returns provides advantage under high uncertainty, Phys. Rev. Research 2, 043174 (2020).

[15] B. Besga, A. Bovon, A. Petrosyan, S. N. Majumdar, and S. Ciliberto, Optimal mean first-passage time for a Brownian searcher subjected to resetting: Experimental and theoretical results, Phys. Rev. Research 2, 032029(R) (2020).

[16] D. Gupta, C. A. Plata, A. Kundu, and A. Pal, Stochastic resetting with stochastic returns using external trap, J. Phys. A 54, 025003 (2020).

[17] M. Magoni, S. N. Majumdar, and G. Schehr, Ising model with stochastic resetting, Phys. Rev. Research 2, 033182 (2020).

[18] M. R. Evans, S. N. Majumdar, and G. Schehr, Stochastic resetting and applications, J. Phys. A 53, 193001 (2020).

[19] A. Miron and S. Reuveni, Diffusion with local resetting and exclusion, Phys. Rev. Research 3, L012023 (2021).

[20] W. Wang, A. G. Cherstvy, H. Kantz, R. Metzler, and I. M. Sokolov, Time-averaging and emerging nonergodicity upon resetting of fractional Brownian motion and heterogeneous diffusion processes, Phys. Rev. E 104, 024105 (2021).

[21] T. Sandev, V. Domazetoski, L. Kocarev, R. Metzler, and A. V. Chechkin, Heterogeneous diffusion with stochastic resetting, J. Phys. A: Math. Theor. 55, 074003 (2022). 
[22] D. Vinod, A. G. Cherstvy, W. Wang, R. Metzler, and I. M. Sokolov, Nonergodicity of reset geometric Brownian motion, Phys. Rev. E 105, L012106 (2022).

[23] A. Pal, S. Kostinski, and S. Reuveni, The inspection paradox in stochastic resetting, J. Phys. A: Math. Theor. 55, 021001 (2022).

[24] J. Fuchs, S. Goldt, and U. Seifert, Stochastic thermodynamics of resetting, Europhys. Lett. 113, 60009 (2016).

[25] D. Gupta, C. A. Plata, and A. Pal, Work Fluctuations and Jarzynski Equality in Stochastic Resetting? Phys. Rev. Lett. 124, 110608 (2020).

[26] S. Reuveni, M. Urbakh, and J. Klafter, Role of substrate unbinding in Michaelis-Menten enzymatic reactions? Proc. Natl. Acad. Sci. USA 111, 4391 (2014).

[27] T. Rotbart, S. Reuveni, and M. Urbakh, Michaelis-Menten reaction scheme as a unified approach towards the optimal restart problem? Phys. Rev. E 92, 060101(R) (2015).

[28] J. M. Meylahn, S. Sabhapandit, and H. Touchette, Large deviations for Markov processes with resetting? Phys. Rev. E 92, 062148 (2015).

[29] P. Singh and A. Pal, Extremal statistics for stochastic resetting systems? Phys. Rev. E 103, 052119 (2021).

[30] Y. Scher and S. Reuveni, A Unified Approach to Gated Reactions on Networks? Phys. Rev. Lett. 127, 018301 (2021).

[31] F. H. Gonzalez, A. P. Riascos, and D. Boyer, Diffusive transport on netwoarks with stochastic resetting to multiple nodes? Phys. Rev. E 103, 062126 (2021).

[32] S. N. Majumdar and G. Oshanin, Spectral content of fractional Brownian motion with stochastic reset? J. Phys. A 51, 435001 (2018).

[33] S. N. Majumdar, S. Sabhapandit, and G. Schehr, Random walk with random resetting to the maximum position, Phys. Rev. E 92, 052126 (2015).

[34] S. N. Majumdar, S. Sabhapandit, and G. Schehr, Dynamical transition in the temporal relaxation of stochastic processes under resetting, Phys. Rev. E 91, 052131 (2015).

[35] J. Masoliver, Telegraphic processes with stochastic resetting, Phys. Rev. E 99, 012121 (2019)

[36] J. Masoliver and M. Montero, Anomalous diffusion under stochastic resettings: A general approach, Phys. Rev. E 100, 042103 (2019).

[37] M. Dahlenburg, A. V. Chechkin, R. Schumer, and R. Metzler, Stochastic resetting by a random amplitude, Phys. Rev. E 103, 052123 (2021).

[38] A. S. Bodrova and I. M. Sokolov, Resetting processes with noninstantaneous return, Phys. Rev. E 101, 052130 (2020).

[39] A. Pal, L. Kusmierz, and S. Reuveni, Invariants of motion with stochastic resetting and space-time coupled returns, New J. Phys. 21, 113024 (2019).

[40] O. Tal-Friedman, A. Pal, A. Sekhon, S. Reuveni, and Y. Roichman, Experimental realization of diffusion with stochastic resetting, J. Phys. Chem. Lett. 11, 7350 (2020).

[41] R. Metzler and J. Klafter, The random walk's guide to anomalous diffusion: A fractional dynamics approach, Phys. Rep. 339, 1 (2000).

[42] S. Burov, J.-H. Jeon, R. Metzler, and E. Barkai, Single particle tracking in systems showing anomalous diffusion: The role of weak ergodicity breaking? Phys. Chem. Chem. Phys. 13, 1800 (2011).
[43] I. M. Sokolov, Models of anomalous diffusion in crowded environments, Soft Matter 8, 9043 (2012).

[44] R. Metzler, J.-H. Jeon, A. G. Cherstvy and E. Barkai, Anomalous diffusion models and their properties: Non-stationarity, non-ergodicity, and ageing at the centenary of single particle tracking, Phys. Chem. Chem. Phys. 16, 24128 (2014).

[45] A. G. Cherstvy, S. Thapa, C. E. Wagner, and R. Metzler, NonGaussian, non-ergodic, and non-Fickian diffusion of tracers in mucin hydrogels, Soft Matter 15, 2526 (2019).

[46] L. Boltzmann, Ueber die Eigenschaften monocyclischer und anderer damit verwandter Systeme, J. Reine Angew. Math. 98, 68 (1885)

[47] J. Klafter and I. M. Sokolov, First steps in random walks: From tools to applications, Oxford University Press (2011).

[48] B. B. Mandelbrot and J. W. van Ness, Fractional Brownian motions, fractional noises and applications, SIAM Rev. 10, 422 (1968).

[49] A. N. Kolmogorov, Wienersche Spiralen und einige andere interessante Kurven in Hilbertschen Raum, CR (Doklady). Acad. Sci. URSS (NS) 26, 115 (1940).

[50] V. Stojkoski, T. Sandev, L. Kocarev, and A. Pal, Geometric Brownian motion under stochastic resetting: A stationary yet non-ergodic process, Phys. Rev. E 104, 014121 (2021).

[51] L. Defaveri, C. Anteneodo, D. A. Kessler and E. Barkai, Regularized Boltzmann-Gibbs statistics for a Brownian particle in a nonconfining field, Phys. Rev. Research 2, 043088 (2020).

[52] I. Gradshteyn and I. Ryzhik, Table of Integrals, Series, and Products, 7th ed. (Elsevier/Academic Press, Amsterdam, 2007).

[53] A. G. Cherstvy, W. Wang, R. Metzler, and I. M. Sokolov, Inertia triggers nonergodicity of fractional Brownian motion, Phys. Rev. E 104, 024115 (2021).

[54] A. G. Cherstvy, S. Thapa, Y. Mardoukhi, A. V. Chechkin, and R. Metzler, Time averages and their statistical variation for the Ornstein-Uhlenbeck process: Role of initial particle conditions and relaxation to stationarity, Phys. Rev. E 98, 022134 (2018).

[55] W. Wang, A. G. Cherstvy, A. V. Chechkin, S. Thapa, F. Seno, X Liu, and R. Metzler, Fractional Brownian motion with random diffusivity: Emerging residual nonergodicity below the correlation time, J. Phys. A 53, 474001 (2020).

[56] H. Safdari, A. G. Cherstvy, A. V. Chechkin, F. Thiel, I. M. Sokolov, and R. Metzler, Quantifying the non-ergodicity of scaled Brownian motion, J. Phys. A 48, 375002 (2015).

[57] A. Bodrova, A. V. Chechkin, A. G. Cherstvy, and R. Metzler, Quantifying non-ergodic dynamics of force-free granular gases, Phys. Chem Chem. Phys. 17, 21791 (2015).

[58] A. G. Cherstvy and R. Metzler, Ergodicity breaking, ageing, and confinement in generalized diffusion processes with position and time dependent diffusivity, J. Stat. Mech. (2015) P05010.

[59] J. H. P. Schulz, E. Barkai, and R. Metzler, Aging Effects and Population Splitting in Single-Particle Trajectory Averages, Phys. Rev. Lett. 110, 020602 (2013).

[60] J. H. P. Schulz, E. Barkai, and R. Metzler, Aging Renewal Theory and Application to Random Walks, Phys. Rev. X 4, 011028 (2014).

[61] R. Hou, A. G. Cherstvy, R. Metzler, and T. Akimoto, Biased continuous-time random walks for ordinary and equilibrium 
cases: Facilitation of diffusion, ergodicity breaking and ageing, Phys. Chem. Chem. Phys. 20, 20827 (2018).

[62] A. G. Cherstvy, H. Safdari, and R. Metzler, Anomalous diffusion, nonergodicity, and ageing for exponentially and logarithmically time-dependent diffusivity: Striking differences for massive versus massless particles, J. Phys. D 54, 195401 (2021).
[63] V. Stojkoski, T. Sandev, L. Kocarev, and A. Pal, Autocorrelation functions and ergodicity in diffusion with stochastic resetting, J. Phys. A: Math. Theor. (2022), doi:10.1088/1751-8121/ac4ce9.

[64] A. G. Cherstvy, O. Nagel, C. Beta, and R. Metzler, NonGaussianity, population heterogeneity, and transient superdiffusion in the spreading dynamics of amoeboid cells, Phys. Chem. Chem. Phys. 20, 23034 (2018). 